\title{
Computer-Based Assessment of Body Image Distortion in Anorexia Nervosa Patients
}

\author{
Daniel Harari ${ }^{1}$, Miriam Furst ${ }^{1}$, Nahum Kiryati ${ }^{1}$, Asaf Caspi ${ }^{2}$, and \\ Michael Davidson ${ }^{2}$ \\ 1 Dept. of Electrical Engineering - Systems, Tel Aviv University \\ Ramat Aviv 69978, Israel \\ \{danielh, mira, nk\}@eng.tau.ac.il \\ 2 Dept. of Psychiatry and Eating Disorders Center, Sheba Medical Center \\ Tel Hashomer 52621, Israel
}

\begin{abstract}
A computer-based method for the assessment of body image distortions in anorexia nervosa and other eating-disordered patients is presented. At the core of the method is a realistic pictorial simulation of lifelike weight-changes, applied to a real source image of the patient. The patients, using a graphical user interface, adjust their body shapes until they meet their self-perceived appearance. Measuring the extent of virtual fattening or slimming of a body with respect to its real shape and size, allows direct, quantitative evaluation of the cognitive distortion in body image. In a preliminary experiment involving 20 anorexia-nervosa patients, $70 \%$ of the subjects chose an image with simulated visual weight gain of about $20 \%$ as their "real" body image. None of them recognized the original body image, thus demonstrating the quality of the transformed images. The method presented can be applied in the research, diagnosis, evaluation and treatment of eating disorders.
\end{abstract}

\section{Introduction}

Anorexia nervosa (AN) is a psychiatric disorder, characterized by low body weight (less than $85 \%$ of the optimal weight), refusal to gain weight, an intense fear of gaining weight or becoming obese, amenorrhea (absence of the monthly period) and perceptual distortions or disturbances of body image or weight [1]. Disordered persons may also exhibit the practice of ingesting large quantities of food and then voiding it through self-induced vomiting, also known as bulimia. Bulimia depletes the body of fluids and of potassium, adversely affecting heart function. The disorder, therefore, involves high incidence of both mental and physical morbidity and high rates of mortality (5-18\% of known AN patients) [18].

Body image distortion is a major symptom of AN and other eating disorders. It is relatively resistant to treatment and can be perceived as a cognitive and perceptual core of the disorder [20]. The assessment of body image is, therefore, a central and reliable diagnostic measure, and is also important for the assessment of the changes during treatment [21]. Since the perceptual distortion allows a 
reliable representation of the pathology, a way of documenting and quantifying the perceptual distortion is of major necessity [30]. Evaluating the existence and severity of the distortion as a direct measure for the perceptual pathology, is also important for research purposes. It may contribute to the understanding of the etiology and the cognitive basis of the disorder, to the evaluation of different treatment methods and to research on the epidemiology of the disorder. For instance, quantifying the distortion in body image at different time points along the course of treatment will give a valid indication for the efficiency of the treatment used. A sensitive measure of body image distortions may be used for early detection, where a clinical intervention may avoid potentially chronic effects of adapting to eating disorders habits (as in anorexia and bulimia nervosa) and other cases of sub-clinical eating disorders.

The increasing incidence of eating disorders and the rising acknowledgment of the central role of the perceptual distortion of the body, lead to the development of various methods for the assessment of body size self-estimation in AN. The first to be employed was the movable caliper or visual size estimation method, which involved the manual manipulation of the distance between two markers until it was perceived to match the particular body part size to be estimated [24]. Various methods, based on the same principle of estimating body part widths, are referred to as body part or body-site methods $[2,22,25]$. One drawback of these methods is that body parts are considered in isolation and the context of the whole body is missing. Furthermore, in these methods an external mediator is involved in conducting the measurements. This can adversely affect the reliability of quantitative measurement of the subject's self-perception and self-assessment.

Other methods focus on estimating the body as a whole $[3,15,13,19]$. In these procedures, the participant views an image and adjusts its size until it matches his or her view of his or her body size [14]. Among the most commonly used procedures is the distorting mirror in which an adjustable mirror distorts the size of the whole body both vertically and horizontally [29]. These whole body distortion methods lead, in most cases, to unnatural, unrealistic images. The distortion level is technically limited and the distortion of specific body parts cannot be controlled.

Computer-based techniques that involve body part and whole body distortions were presented in $[12,23]$. Both methods provide body outlines or silhouettes, not a realistic recognizable image of the participant.

In this paper we present a new diagnostic tool that facilitates evaluation of the self perception of the subject's body image and provides a quantitative measure of body image distortion. Starting with a real image of the subject, highly realistic fattened and slimmed images are synthesized. Body parts are independently modified while maintaining the natural appearance and global characteristics of the body shape. User interactivity is simple and friendly, allowing direct manipulation by an untrained subject without an external mediator. 


\section{The Algorithm for Weight-Change Simulation}

Fattening and slimming processes have different effects on different body parts. The body weight-change simulation algorithm first subdivides the body image into principal body-parts, that have different patterns of shape-change, when a weight gain or loss process takes place. Each body part is separately and independently processed. Then, the altered body parts are merged back into a whole body, with special attention to joint areas. The whole process is based on a method developed for the representation of biological shape modifications (such as growth and evolution) in living organisms.

\subsection{Image Acquisition and Preprocessing}

The subjects (eating disorders patients) are dressed in pink or purple colored full-body leotards. Front-view body images are captured with a Sony digital still camera (model MVC-FD73), with the subjects' arms raised by about $25^{\circ}$, having their fists clenched and their legs parted by about $50 \mathrm{~cm}$. A uniform matte blue screen is used as background. The pixel resolution is $640 \mathrm{x} 480$.

The identification of body parts is semi-automatic. The user points at seven different landmarks on the source body image. Slightly misplaced landmarks are corrected automatically. These seven landmarks are used by the algorithm to isolate ten different body parts (left hand, right hand, head, upper chest, lower chest, belly, hips, pelvis, left leg, and right leg). Each body part is processed independently, according to its shape-change characteristics.

The extraction of the body figure from the background of the image is automatic. First the image is transformed from the RGB color space to the HSV (Hue, Saturation, brightness Value) color space. Since the background is uniform matte blue, hue-thresholding (supported by an adaptive brightness threshold for reliable segmentation of hair) allows sharp and exact extraction of the body figure from the source image (see the middle column in Fig. 1). The segmented body shape, together with the seven user marked landmarks, is used to create a binary mask for each of the ten different body parts. These masks are essential for the shape transformation algorithm, which is applied to each body part independently.

\subsection{The Method of Transformation Grids}

There exist several techniques and methods for the representation of shape and shape change. However, most of these techniques refer to shapes as still objects and to shape changes as partial displacements governed by external forces. Since in our case the shapes to be modified are human body parts, that have their own pattern of growth and deformation, a special kind of shape change method is required. Such a method was presented by Bookstein [6] who has found, in his research, a way to express biological shape differences using a mathematical approach based on D'Arcy Thompson's distorted grid graphs [28]. 
Thompson invented the method of transformation grids to represent the relationship between a pair of homologous shapes throughout their interiors. A homology between two organisms is defined to be a "maximally inclusive scheme of pairs of parts, organs or structures that manifest the same positional relations among themselves in both organisms" [6]. Thompson has drawn grid intersections at roughly homologous points on two organisms, and then showed how the square grid for one was distorted to produce the grid of the other. He suggested to interpret the pair of diagrams as a transformation of the whole picture plane which maps the points of one diagram into corresponding points in the other, while varying smoothly in between. His suggestion accorded with his belief that constituent parts of an organism could never evolve quite independently [6].

While exploring a mathematical model to produce Thompson's distorted grids, Bookstein [6] argued that Thompson was wrong in the construction of pairs of diagrams which were unsymmetrically specified: a rectangular grid on one diagram and an unrestricted grid on the other. Instead, Bookstein suggested the use of biorthogonal grids for the transformation as a representation of general lines of growth. He produced a canonical coordinate system which reduces all change of shape to gradients of differential directional growth [6], enabling the measure of shape change without measuring shape at all. In computing the biorthogonal curve systems, Bookstein assumed correspondence between boundaries and extended it to the interiors. His strategy was to describe the homology between two images by discrete pairs of homologous landmarks. He chose a complex biharmonic function, with only isolated "sources" and "sinks" of distortion as a convenient model for the homologous correspondence between the two images, from which the mapping function could be defined. Considering a mapping of a small patch, Bookstein defined the roughness of the transformation as the squared distance between the mapping of the centroid of the patch and the centroid of the mapped patch. Further, he has shown that if the roughness of the transformation is minimized, the non-linear mapping function will be uniquely defined, and the biological characteristics of the shape will be preserved $[6,7,8]$.

Formally, suppose we are given a shape $\mathcal{P}$ that should be transformed into a shape $\mathcal{Q}$. Let $\left\{P_{i}=\left(X_{i}, Y_{i}\right)\right\}$ be a subset of $N$ points on the outline of $\mathcal{P}$, and suppose that their corresponding points on the outline of $\mathcal{Q}$ are respectively $\left\{Q_{i}=\left(U_{i}, V_{i}\right)\right\}$. Based on these homologous (corresponding) pairs, Bookstein [6] defines the following transformation from any point $P=(X, Y) \in \mathcal{P}$ to a point $Q=(U, V) \in \mathcal{Q}:$

$$
\begin{aligned}
& U=a_{u} \cdot X+b_{u} \cdot Y+c_{u}+\sum_{i=1}^{N} \omega_{i}^{u} \cdot F\left\{\delta\left(Q_{i}, P\right)\right\} \\
& V=a_{v} \cdot X+b_{v} \cdot Y+c_{v}+\sum_{i=1}^{N} \omega_{i}^{v} \cdot F\left\{\delta\left(Q_{i}, P\right)\right\},
\end{aligned}
$$

where $\delta\left(Q_{i}, P\right)$ is the Euclidean distance between $Q_{i}$ and $P$ and

$$
F(\delta) \equiv \delta^{2} \cdot \log \left(\delta^{2}\right) .
$$


This transformation is parameterized by $2 N+6$ parameters, namely $a_{u}, b_{u}$, $c_{u},\left\{\omega_{i}^{u}\right\}_{i=1}^{N}, a_{v}, b_{v}, c_{v},\left\{\omega_{i}^{v}\right\}_{i=1}^{N}$. The transformation can be made unique by requiring that its roughness be minimized, where the roughness $R$ is defined as

$$
R=\iint\left[\left(\nabla^{2} U\right)^{2}+\left(\nabla^{2} V\right)^{2}\right] d U d V
$$

Differentiating $R$ and equating to zero, leads to the following six constraints on the parameters:

$$
\begin{aligned}
& \sum_{i=1}^{N} \omega_{i}^{u}=\sum_{i=1}^{N} \omega_{i}^{v}=0 \\
& \sum_{i=1}^{N} \omega_{i}^{u} \cdot U_{i}=\sum_{i=1}^{N} \omega_{i}^{v} \cdot U_{i}=0 \\
& \sum_{i=1}^{N} \omega_{i}^{u} \cdot V_{i}=\sum_{i=1}^{N} \omega_{i}^{v} \cdot V_{i}=0
\end{aligned}
$$

Now, substituting the $N$ corresponding pairs $P_{i} \rightarrow Q_{i}$ in Eqs. 1 and Eqs. 3 yields $2 N+6$ equations from which the unknown parameters can be obtained. Using these parameters, the transformation given by Eqs. 1 can now be applied to all points $P \in \mathcal{P}$.

\subsection{Body Shape Transformation}

The outcome of the preprocessing steps (subsection 2.1) are a segmented body image and the shapes of the ten body parts represented by binary masks. In order to simulate weight-change in each body part according to Bookstein's model, there is a need for pairs of corresponding landmarks in the original and modified shapes.

For each body part, a set of three arbitrary points $P_{i}=\left(X_{i}, Y_{i}\right)$, located far from each other on the outline, is selected. These three points are mapped to points $Q_{i}=\left(U_{i}, V_{i}\right)$ on the outline of the modified shape by scaling the magnitudes of the vectors connecting them to the centroid. The scaling factor is is specific to the body part and proportional to the global change required.

Substituting for the three pairs of points $P_{i} \rightarrow Q_{i}$ and for $N=3$ in Eqs. 1 and Eqs. 3, yields 12 independent linear equations, from which the coefficients $a_{u}, b_{u}$, $c_{u},\left\{\omega_{i}^{u}\right\}_{i=1}^{3}, a_{v}, b_{v}, c_{v}$, and $\left\{\omega_{i}^{v}\right\}_{i=1}^{3}$ can be obtained. Using these coefficients, Eqs. 1 are now used to transform the complete outline of the original body part into a weight modified outline. Repeating this procedure for all body parts, yields a set of independently transformed outlines.

The next step is to merge the modified outlines of all the body-parts into a single outline of a whole body. Adjacent pairs of body parts are merged, subject to constraints that preserve the natural appearance of the modified body shape and global characteristics such as body height and skeleton integrity. 
Having obtained the whole outline of the weight-modified body, $N$ pairs of corresponding points from the original and modified outlines are selected $(N$ is about $10 \%$ of the total number of points on the outline). Substituting for these pairs of points in Eqs. 1 and Eqs. 3, we obtain $2 N+6$ equations that uniquely define the coefficients in Eqs. 1. These coefficients specify the transformation of the whole body. Eqs. 1 can now map all points $(X, Y)$ in the original body image onto points $(U, V)$ in the weight modified body image.

The ratio between the $2-\mathrm{D}$ body areas in the modified and original images is a useful quantitative index for body image distortion evaluation. Using a cylindric approximation for the 3-D structure of body parts, weight ratio is roughly the square of the area ratio. A better approximation could be obtained by monitoring the weight and 2-D body-shape area of humans during weight change processes.

\subsection{Postprocessing}

The non-linear transformation maps each point in the original body image onto the target image plane. Due to discretization effects, some points in the modified body image have no corresponding source points in the original image, and therefore lack color properties. These discontinuities are eliminated using $3 \times 3$ median-based color interpolation around colorless pixels. This procedure may create some artifacts on the external side of the body outline. These are masked using the (known) modified outline. The result is a clear, sharp and realistic color body image, in which the body figure is fatter or slimmer by the specified weight-change factor. Typical results are shown in Fig. 1.

\section{Implementation and Performance Evaluation}

The suggested method for the assessment of body image distortions in eatingdisordered patients is implemented in the MATLAB environment, and is executable on computers with a wide variety of operating systems. For each subject, a set of weight-modified body images is generated offline and stored as a virtual album. A graphical user interface is provided for controlling and monitoring the automated body-image album generation.

Convenient access to the stored images is facilitated by a graphical browser, allowing immediate retrieval and display of the weight-modified body images.

To evaluate the quality of the weight-modified images, an experiment was conducted among 30 healthy people (not suffering from eating disorders). The purpose of the experiment was to determine whether the simulated body images were realistic enough, to confuse an original body image with an artificially generated one.

The experiment consisted of 45 trials. In each two body images were presented: an original image and an artificial one. A total of 15 simulated image albums of teenage and young female adults were used in the experiment. The weight change factor of the simulated image in each trial was chosen in the range $\{-16 \%,+28 \%\}$. The position (right/left) of the two images on the screen 

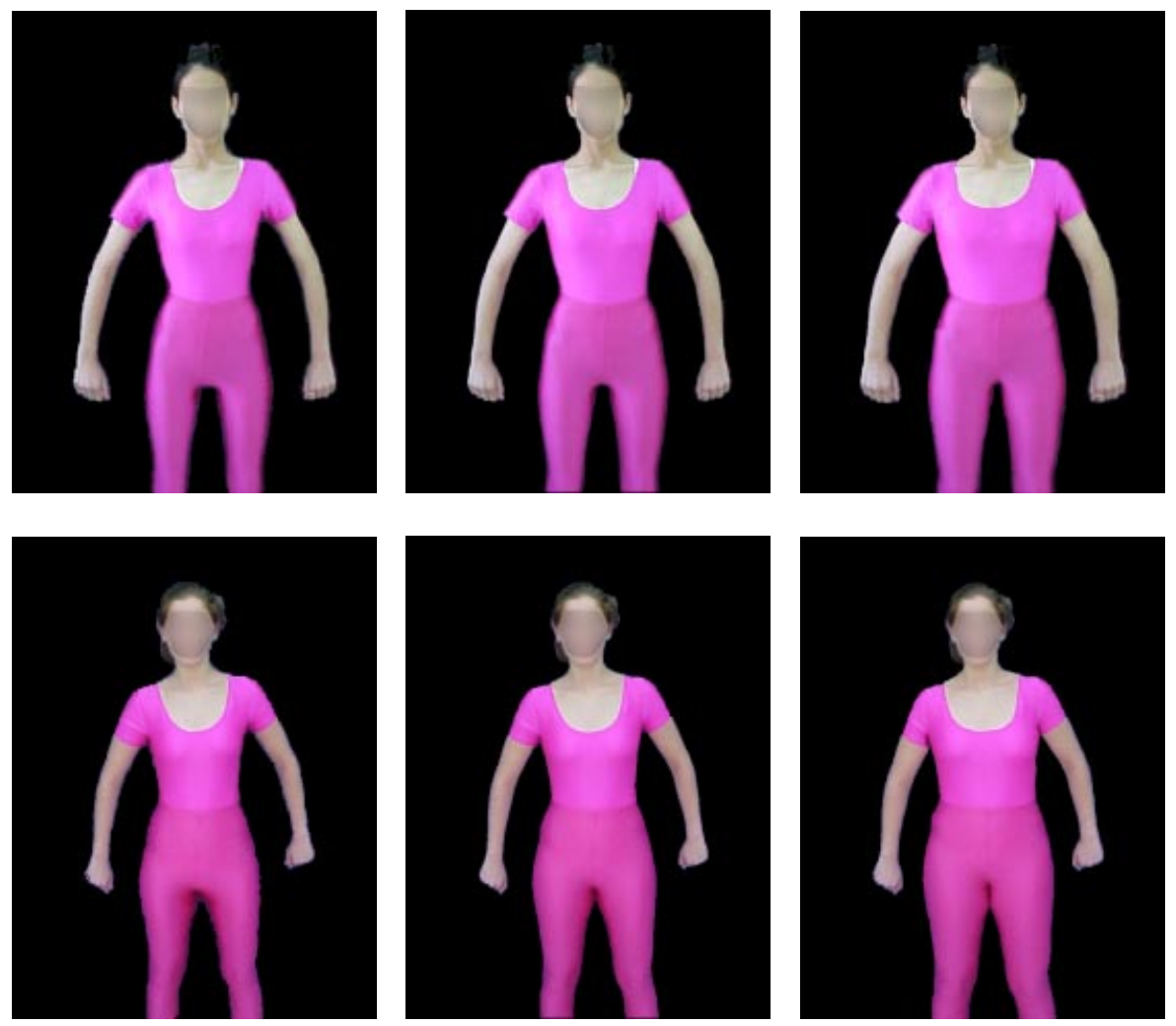

Fig. 1. Middle column: Body images of two anorexia-nervosa patients, extracted (segmented) from the original images (not shown). Left column: Computer generated body images with about $5 \%$ weight loss. Right column: Computer generated body images with about $15 \%$ weight gain.

was random. In each trial the subject was asked to choose which of the two displayed images was the original body image. A graphical user interface was used to control the experiment and store its results automatically.

The results of the experiment show that in trials with a simulated weight change of up to ${ }_{-}^{+} 8 \%$, the subjects confusingly chose the artificial body image as the "original" one in $44 \%$ of the cases. This proves that the artificially generated body images are highly realistic. As could be expected, larger simulated weight changes appear less natural and are easier to detect. Over the full weight-change range of $\{-16 \%,+28 \%\}$ the error rate of subjects was about $30 \%$. 


\section{Discussion}

We presented a method for the assessment of body image distortions in eating disorders patients, that improves upon previous techniques. Realistic simulation of the human body weight-change process is achieved via independent manipulation of separate body parts, followed by the presentation of the subject's natural-looking weight-modified body image.

The weight change simulation algorithm is based on a unique biological shape modification technique presented by D'Arcy Thompson and Bookstein $[28,6]$. Other methods for shape deformation and modification do exist. Snakes or active contour models are energy-minimizing splines guided by external constraint forces and influenced by image forces that pull them toward features such as lines and edges [16]. Some active-contour based models suggest shape deformation by making the shape's contour behave like a balloon which is inflated by an additional force [10]. An active contour model with expansion "balloon" forces was used to simulate the changes in shape and cross-sectional area occurring during the contraction of isolated muscle fibre [17].

Caricaturing algorithms $[9,26]$ exaggerate features on the shape's contour. The resulting shapes resemble the original ones but are not natural looking. Image morphing techniques [11,27] are defined between at least two shapes or objects. However, in the case of weight change simulation only the original body image is given, hence we remain with the problem of creating a synthesized target body image to which the metamorphosis process can be targeted.

Based on facial image caricaturing [5], an interesting method for the study of body size perception and body types was very recently presented [4]. Using clinical categories of body types (BMI - Body Mass Index) as prototypes, two approaches to changing the body shape were considered. The first approach alters the body shape by exaggerating or minimizing differences of feature points on the original body and its corresponding prototype. The second approach alters the body shape by referring to all prototypes simultaneously, following evolutionary paths of feature points among the prototypes. A large image-database (hundreds of images) is necessary for the extraction of stable prototypes with minimal variability within each prototype and with similarity between the BMI category prototypes [4]. The user is required to manually delineate hundreds of feature points, used by the system to locate and extract the body and the different body parts, and to refer to the category prototypes.

The method presented here was developed as the first phase of a two-phase project. In the next phase of the project, the body-image distortion as assessed by the method presented, will be compared with the distortion as assessed by several common and clinically validated assessment tools, such as: (a) structured interview of anorexia and bulimia syndromes, (b) eating disorder inventory, and (c) body shape questionnaire. In addition, the algorithm's assessment results will be compared to common clinical measures of diagnosis and follow-up: (a) body weight and BMI, (b) menstrual cycle, and (c) stage of therapy.

The proposed body image distortion assessment tool is currently being deployed and tested at the Eating Disorder Center of Sheba Medical Center. A 
group of 20 admitted patients suffering from AN participated in an experiment, in which they where asked to choose an image from a 24-picture album of their body (at various simulated weight-change levels), that corresponds to their body size as they perceive it. A high percentage of the subjects (70\%), both youth and young adults, chose an image in which a visual weight gain of about $20 \%$ was simulated, as their "real" body image. None of them recognized their true source body image. This demonstrates the quality of the transformed body images. The suggested method is expected to be a valuable tool for diagnosis, treatment and follow-up in patients with eating disorders.

\section{References}

1. American Psychiatric Association (1994). "Diagnostic and Statistical Manual of Mental Disorders", 4th ed.

2. Askevold F. (1975). "Measuring Body image". Psychotherapy and Psychosomatics, Vol 26, pp. 71-77.

3. Bell C., Kirkpatrick S. W., \& Rinn R. C. (1986). "Body image of anorexic, obese, and normal females". Journal of Clinical Psychology, Vol. 42, pp. 431-439.

4. Benson P. J., Emery J. L., Cohen-Tovee E. M., \& Tovee M. J. (1999). "A comutergraphic technique for the study of body size perception and body types". Behavior Research Methods, Instruments $\&$ Computers, Vol. 31, pp. 446-454.

5. Benson P. J., \& Perrett D. J. (1991). "Synthesizing continuous-tone caricatures". Image EG Vision Computing, Vol. 9, pp. 123-129.

6. Bookstein F. (1978). "The measurement of biological shape and shape change". Lecture Notes in Biomathematics, Springer-Verlag New York.

7. Bookstein F. L., \& Green W. D. K. (1993). "A feature space for derivatives of deformations". Information Processing in Medical Imaging, 13th International Conference, IPMI '93 Proceedings, Springer-Verlag, Berlin, Germany, pp. 1-16.

8. Bookstein F. L. (1989). "Principal warps: Thin plate splines and the decomposition of deformations". IEEE Transactions on Pattern Analysis 83 Machine Intelligence, Vol. 11, pp. 567-585.

9. Brennan S. E. (1985). "Caricature generator: The dynamic exaggeration of faces by computer." Leonardo, Vol. 18, pp. 170-178.

10. Cohen L. D. (1991). "On active contour models and balloons". CVGIP - Image Understanding, Vol. 53, pp. 211-218.

11. Daw-Tung L., \& Han H. (1999). "Facial expression morphing and animation with local warping methods". Proceedings 10th International Conference on Image Analysis and Processing, IEEE Computer Society, Los Alamitos, CA, USA, pp. 594-599.

12. Dickson-Parnell B., Jones M., Braddy D., \& Parnell C. P. (1987). "Assessment of body image perceptions using a computer program". Behavior Research Methods, Instruments, \& Computers, Vol. 19, pp. 353-354.

13. Freeman R. J., Thomas C. D., Solyom L., \& Hunter M. A. (1984). "Video-camera for measuring body image distortion". Psychological Medicine, Vol. 14, pp. 411-416.

14. Gardner R. M. (1996). "Methodological issues in assessment of the perceptual component of body image disturbance". British Journal of Psychology, Vol. 87, pp. 327-337.

15. Garner D. M., Garfinkel P. E., \& Bonato D. P. (1987). "Body image measurement in eating disorders". Advances in Psychosomatic Medicine, Vol. 17, pp. 119-133. 
16. Kass M., Witkin A., \& Terzopoulos D. (1987). "Snakes: active contour models". International Journal of Computer Vision, Vol. 1, pp. 321-331.

17. Klemencic A., Pernus F., \& Kovacic S. (1999). "Modeling morphological changes during contraction of muscle fibres by active contours". Computer Analysis of Images and Patterns, 8th International Conference, CAIP'99. Proceedings, SpringerVerlag, Berlin, Germany. (Lecture Notes in Computer Science, Vol. 1689, pp. 134141).

18. Neumaker K. J. (1997). "Mortality and sudden death in anorexia nervosa". International Journal of Eating Disorders, Vol. 21, pp. 205-212.

19. Probst M., Vandereycken W., \& Van Coppenolle H. (1997). "Body size estimation in eating disorders using video distortion on a life-size screen". Psychotherapy 86 Psychosomatics, Vol. 66, pp. 87-91.

20. Rosen J. C. (1990). "Body image disturbance in eating disorders". in T. F. Cash: Body images: development, deviance and changes, New York: Guilford, pp. 190-214.

21. Rosen J. C. (1996). "Body image assessment and treatment in controlled studies of eating disorders". International Journal of Eating Disorders, Vol. 20, pp. 331-343.

22. Ruff G. A., \& Barrios B. A. (1986). "Realistic assessment of body image". Behavioral Assessment, Vol. 8, pp. 235-251.

23. Schlundt D. G., \& Bell C. (1993). "Body image testing system: a microcomputer program for assessing body image". Journal of Psycho-pathology \&5 Behavioural Assessment, Vol. 15, pp. 264-285.

24. Slade P. D., \& Russell G. F. M. (1973). "Awareness of body dimensions in anorexia nervosa: Cross-sectional and longitudinal studies". Psychological Medicine, Vol. 3, pp. 188-199.

25. Thompson J. K., \& and Spana R. E. (1988). "The adjustable light beam method for the assessment of size estimation accuracy: Description, psychometrics and normative data". International Journal of Eating Disorders, Vol. 7, pp. 521-526.

26. Steiner A. (1995). "Planar shape enhancement and exaggeration". M.Sc. thesis, Department of Electrical Engineering, Technion - Israel Institute of Technology, Haifa, Israel.

27. Tal A., \& Elber G. (1999). "Image morphing with feature preserving texture". Blackwell Publishers for Eurographics Association. Computer Graphics Forum, Vol. 18, pp. 339-348.

28. Thompson D. W. (1917). "On growth and form" (abridged edition, 1966). Cambridge University Press, London, Great Britain.

29. Traub A. C., \& Orbach J. (1964). "Psychophysical studies of body image. I. The adjustable body-distorting mirror". Archives of General Psychiatry, Vol. 11, pp. 53-66.

30. Williamson D. A., Cubis B. A., \& Gleaves D. H. (1993). "Equivalence of body image disturbances in anorexia and bulimia nervosa". Journal of Abnormal Psychology, Vol. 102, pp. 177-180. 